\title{
Theory of the Evolution of Nurse Practitioner Practice
}

NPOJ 2021, 1, pp. 23-36

Roberta Heale, DNP, PhD, NP-PHC

rheale@laurentian.ca

1. Laurentian University, Sudbury, Ontario, Canada

\begin{abstract}
Aim: To determine the nurse practitioner's perception of scope of practice and understanding of changes to practice over time.

Background: A great deal of research about nurse practitioners has been conducted related to such things as role clarity, transition, or preparedness to practice and job satisfaction. Conceptual models of nurse practitioner practice have been developed to highlight practice processes, interprofessional relationships and more. However, none of this literature addresses nurse practitioner's perception of scope of practice, the impact of their changing practice experiences and how the understanding of their practice changes over time.

Methods: This grounded theory study was undertaken in Ontario, Canada, with interviews of primary health care nurse practitioners which resulted in the Theory of the Evolution of Nurse Practitioner Practice. Conclusion: Within this theory, scope of practice is defined as nurse practitioners working to their maximum potential. There are both intrinsic and extrinsic elements which render maximum practice potential a fluid and changing state.
\end{abstract}

\section{Introduction}

Nurse practitioner (NP) practice has been described as the integration of the expertise of two health disciplines, nursing and medicine, a state which renders NP work to be unique and complex (Shuler \& Davis, 1993b). A great deal has been studied and written about NPs and their work, however, there still is no one definition of scope of practice. In addition, although much has been learned about NP roles and transition to practice, there remains a gap in the understanding of how NP practice grows and changes over time. This was explored in a grounded theory study of primary health care NPs from Ontario, Canada.

\section{Literature Review: Scope of Practice}

Much of the discussion about NPs focuses on them working 'to their full scope of practice', but the meaning of this isn't always clear. Scope of practice is often discussed as a list of tasks (diagnose, prescribe, order tests, etc.) and, in this case, it is a term used to describe the regulated functions that NPs are authorized to perform. Thus, one 
definition of scope of practice is that it is "a set of rules, regulations, and boundaries within which a fully qualified NP may practice" (Kleinpell, Hudspeth, Scodo \& Magdic, 2012, p. 12). Scope of practice may also be referred to as competencies, or standards (Miller, 2020). Yet, legislative requirements don't always reflect the nature of NP practice.

The concept of scope of practice is broader than individual controlled acts, standards, credentialing, or authority, and is sometimes referred to as the ability of NPs to work to the full extent of their education and training (Donelan, DesRoches, Dittus \& Buerhaus, 2013). Within this framework, discussion typically centres around limitations to NP practice and the inability to meet their full practice potential (Kleinpell, et al., 2012; Donelan, et al., 2013). For example, in a study in the USA a group of NPs were surveyed and asked to respond to questions about their practice. The NPs in the study indicated that they were not working to their full potential as a result of practice restrictions that limited their ability to provide full primary health care services to patients and to be the primary care provider, or most responsible provider for patients (Donelan, et al., 2013).

Discussion about scope of practice also arises in research about role clarity for NPs. One focus of this literature is on demographics, including the number of, and where NPs practice. Articles often include reflections on the perception of other health care providers and the community at large in the understanding of NPs in practice in primary health care, acute care or other health care settings (Donald et al, 2010; Kilpatrick et al., 2010). Further reviews of the role of NPs include analysis of their work in relation to other health care team members (Hurlock- Chorostecki, et al., 2014). This body of literature examines how the view of the NP's role held by NPs and others, impacts their practice.

NP practice is also targeted in conceptual models. One of the best known is the Shuler Nurse Practitioner Practice Model. This theoretical model outlines the integration of the nursing and medical competencies of the NP and demonstrates the interaction and relationship between them in a conceptual diagram (Shuler \& Davis, 1993a). Although developed in the early 1990's, this model remains one of the foremost conceptual descriptions of NP practice. However, it doesn't provide insight into the factors influencing the extent to which NPs integrate each competency into their practice, or how the practice changes over time.

Literature centering on the transition to, and preparedness for practice also includes reflections on NP practice. In this body of work, the level of comfort with various aspects of NP practice authorities is reviewed, including assessment, diagnosis, treatment and more, as well as the barriers and facilitators to achieving a level of comfort in the role (Hart \& Bowen, 2016). Stressors and interprofessional relationships are also discussed (Barnes, 2015). In both these areas, NP practice is largely viewed as the competencies and tasks related to NP work. 
None of these areas: role clarity, conceptual models, transition, or preparedness to practice, accurately address the nuances of NP's perception of scope of practice, or the impact of their changing practice experiences. None of this literature answers the question about what occurs when an NP has completed the transition into practice. In her seminal work which explored the differences between beginning and expert nurses in clinical performance and situational appraisal, Patricia Benner (1984) provides a rich description about nursing practice, including levels of practice (novice to expert) and how they are characterized within various domains of nursing care. The findings are not specific to NPs and it isn't known whether they can be used to explain changes in how NPs practice. However, they offer insight into the fact that nursing practice is a dynamic concept, evolving over time as the provider gains knowledge and practice experiences. It is presumed that NP practice evolves in a similar manner.

\section{Aims}

Historically, NPs have had to put effort into ensuring comprehensive regulation and a wide range of practice opportunities. However, the understanding of NP practice over time has been neglected. This study was undertaken in order to gain a better understanding of changes to NP practice over time. The purpose of this study was to develop a theory about the evolution of NP practice.

\section{Design}

Grounded theory allows the researcher to move beyond description to the generation of a unified theoretical explanation of a phenomenon (Creswell, 2013). In order to determine the specific factors and relationships that are found in the practice of NPs, a grounded theory approach was chosen for this study (Corbin \& Strauss, 2015). Data were collected and analyzed through an iterative process and were used to develop a definition of NP scope of practice and a framework of the evolutionary process of NP practice. Ethical approval for this study was obtained through Laurentian University Research Ethics Board.

Purposive sampling strategies were used to recruit participants (Corbin \& Strauss, 2015). Participants should have enough experience with the phenomenon to adequately describe it (Corbin \& Strauss, 2015). To meet this criteria NPs participating in the study had to have at least a year full time practice in primary health care. NPs who had practiced with the researcher, an NP herself, were not eligible to participate in the study. Although the focus of the interviews was NP practice in primary health care, participants with practice experience in other settings were also included. The heterogeneity of the sample assisted in the confirmation of the theory arising from the data analysis (Creswell, 2013).

\section{Data Collection}

Data were collected through virtual interviews that lasted approximately one hour in duration, with shortest being 37 minutes and the longest 70 minutes. The interviews 
were guided by semi-structured questions which were modified, as required, to provide more detail about emerging concepts (Corbin \& Strauss, 2015). Initial questions were:

1. Describe your practice. Do you have your own roster of patients for whom you are the primary care provider?

2. Have you practiced in any other primary health care organizations? If so, was your practice different and how?

3. In your opinion you work to your full scope of practice? Please Describe.

Memos were created during the data collection process and a summary of each interview was created. Each interview was audiotaped and transcribed in its entirety by a research assistant, using the process described in Creswell (2013). Transcripts were double-checked for accuracy by the researcher against the taped file as well as the interview notes.

When the interviews and preliminary data analysis were completed, a summary of the findings was emailed to each participant. The purpose was to request their feedback, to ensure that they reflected their experience as an NP. No changes to the initial findings were requested by the participants at this stage.

\section{Sample}

The number of participants in a sample for a grounded theory research study is guided by the extent to which data saturation occurs; defined as when no new categories, relevant themes, or relationships emerge (Corbin \& Strauss, 2015). In this research thirteen participants were in communication with the researcher. Two did not follow through with the interview. Another did not meet the inclusion criteria. Interviews were conducted with the remaining ten NPs. The data analysis process began with each interview as soon as the transcript was prepared. Concepts quickly emerged from the data and, with no substantially new information emerging in the last three interviews, data collection ended after the tenth interview.

\section{Data Analysis}

Corbin \& Strauss (2015) describe data analysis as process. They view the phenomena as the focus of the research; in this study it is NP practice. They then describe process as the actual things people do to create that phenomenon. In this study the actions, interactions or strategies that NPs undertake, or experience that comprise their practice, are process. Using this tactic, the analyst undertook an interactive role, seeking to understand not only how NPs describe their day-to-day practice, but how they contextualize their experience as NPs.

Detailed analysis of each transcript was conducted to ensure that no important ideas or constructs were missed. Insights were coded and those that were similar conceptually were grouped together as concepts. Using constant comparison, the most relevant concepts were identified and the relationships between them explored. The 
relationship between the concepts was identified and they were then integrated, forming a theoretical framework (Corbin \& Strauss, 2015). The Evolution of NP Practice Theory describes the phenomenon outlined in the purpose of the research and the interactions and relationships related to it.

\section{Findings}

This discussion focuses on the reflections of the NPs on their practice. Details demonstrate and justify the main concepts of the theory.

\section{Maximum Practice Potential}

Since the term scope of practice is used throughout NP literature, the NPs were asked whether they thought they worked to their full scope of practice. In the answers, the participant's description of their scope of practice was as a concept, not as simply as the performance of authorized functions. They described it as a reflection of their personal boundaries of clinical practice, and as a continuum. They noted that it changes during the course of an NP's career. One participant described scope of practice: "I would say that depending on where you work, ... you are meeting your maximum potential." (Participant \#1) Another stated that scope of practice "Is knowing your limits and knowing where to put your own boundaries." (Participant \#7) Yet another indicated: "I would say I'm working to my full scope of practice... like pertaining to my history and my experience um, if that makes sense." (Participant \#9). During the analysis both intrinsic and extrinsic factors were revealed which impact the NP's ability to practice to their maximum potential.

\section{Intrinsic}

\section{Ability to Achieve Maximum Potential}

The breadth of an NP's scope of practice is, in part, related to their own personal initiative. To start, it depends on the level of a NP's personal motivation. "NPs often have to carve their own path...be motivated to learn, grow in the role." (Participant \#4) The participants indicated that this doesn't simply happen and that NPs must purposefully and consciously work toward higher levels of competency and expertise. "So, it's not about, you know, this drug or that drug, or that diagnosis, it's about how I achieve excellence in my role.' (Participant \#10) They are required to challenge themselves and have a "...personal drive to learn new things." (Participant \#8)

I really love the complexity and I love the challenge that it gives me, like, the level of learning is phenomenal. Um, I mean, I'm motivated uh, daily to participate in webinars and, and take online courses and stuff just so I can, you know, manage my day-to-day practice. So, it's really pushed me um, as far as my knowledge goes. And um, I'm never bored; I'm always challenged. (Participant \# 2) 
Several participants indicated that some NPs achieve a certain level of expertise and do not move on: "People who find their niche and they don't want to move out of their niche." (Participant \# 10) However, the importance of continuously growing in the NP role is stressed. "I think we really have to um, ask ourselves, you know, is my job to stay comfortable or is my job to support the client? (Participant \# 10)

Part of achieving maximum practice potential is the development of a personal understanding of what it looks like. This is seen as a reflection on the extent to which NP practice is modeled at maximum potential.

Right, and so I look at these NPs ....and you know they have this little comfortable niche, and the doctors have their little comfortable niche, and nobody is challenging them, right? And nobody is saying look, this is what can be done, isn't this exciting, isn't this where we can be? (Participant \#10).

Working with NPs who have not evolved in their role also has an impact on the practice of an NP who has done so, leaving them without mentorship. "Um, I guess moving here I'm much more independent, so, um, like there's not kind of anybody else I can follow and to sort of explain my clinical reasoning." (Participant \#9)

\section{Maximum Practice Potential is Fluid}

The focus of NP practice changes among different environments so that maximum potential is different in different practices. "Once you become part, once you settle into a practice you can then, um, look for opportunities to challenge yourself further and still meet the specific needs of the population that you work with." (Participant \#3)

Each NP has their own maximum potential, changed by experiences and life circumstances. Through these, the focus area of expertise changes. For example, one of the participants indicated that she'd stopped doing intrauterine device insertion because she changed jobs and doesn't see young women as often and now faces the challenges of practicing with an older, complex population. "As you know older patients have a more complex caseload...My practice is slowly turning over, I actually have some 100 -year old women." (Participant \#7)

\section{Extrinsic}

\section{Facilitators}

Along with the personal factors influencing an NP's ability to achieve maximum practice potential, there are many extrinsic facilitators and barriers that have an impact. One facilitator is being the primary care provider, or most responsible provider to a roster of patients. In this role, the "NP is accountable" and this accountability "...extends the boundaries of clinical potential." (Participant \#9) and "This environment um, is a good 
environment to maximize your scope of practice because you're the primary care provider... you know, responsible for the whole breadth of primary care." (Participant \#9).

You know the beauty of the uh, (states organization), is that you have the freedom to do what you need to do for your patients. If there is a perceived need uh, for your client um, you make it happen. (Participant \#10)

Another facilitator for the NP to be a most responsible provider is appropriate organizational support, otherwise their ability to grow in their practice is stymied. The level of autonomy and organizational support of their day-to-day practice is a facilitator in the growth of their practice.

It just depends on the practice and the day and sort of you know, what each individual provider's capacity may be that day. But, we...I've never felt pressured to see a certain number of patients a day, um, it's generally not an issue... Um, yeah, uh, the admin is very, very supportive of our practice, it's a very positive work environment. (Participant \#5)

and a team that works well together is also supportive:

We do have a clinic assistant that does all the vitals and everything before we see our clients, so all of that kind of stuff is done before they come in, and uh, the nurses do the uh, bulk of the prenatal assessments too so that we're just, um, you know, reviewing the form and doing fundal heights and hearts and so forth to uh... and ordering testing so we have a fairly significant amount of support. Really, it's quite good. (Participant \#5)

Also important to the NP in reaching maximum practice potential is a receptive, collegial organizational environment. In some organizations "NPs work to their potential and are encouraged to do so." (Participant \#5), "The organizational expectation is that every NP will be able to meet full legislative requirements such as prescribing controlled substances." (Participant \#10).

The benefits of NPs in clinical leadership roles were cited. (Participant \#6). On the other hand, NP practice may be limited by interactions with other providers, particularly by some physicians. "She (physician) would have restricted my capacity to grow, yeah absolutely." (Participant \#10).

Support and resources for continuing educational opportunities is also an extrinsic factor in the NP achieving maximum practice potential, as are informal, collegial learning opportunities in their practices.

The NPs we have a monthly...meeting where at time we will debrief a case or um, work through one (a case)...We, the NPs and some of the physicians belong to a 
problem-based learning group...most of us have done project ECHO (online learning forum for clinical practice) (Participant \#6)

So we do a lot of discussion at a round table to review certain individuals to discuss okay they're seeing this person, ...okay how are they doing, what can we change...it's interdisciplinary...it's very informal, like just a phone call away, or a pop down the hallway, saying 'hey can you go look at something for me?' or 'what do you think I should do for this?' (Participant \#3)

\section{Barriers}

\section{Unrealistic Workload Expectations}

Just as there are organizational supports that encourage NPs to achieve their maximum potential in clinical practice, there are many factors that hinder this growth. All of the NPs indicated that their patient panel included a large number of vulnerable people with high socioeconomic need. The NPs pointed out that the complexity with this group of patients was more than for those with only medical issues. The added clinical burden of these complex cases wasn't always recognized by the organization and, in fact, patients with many non-medical issues were often diverted to the NPs.

... it's kind of all ages and stages but quite a marginalized population in terms of income, education, um, employment, all of those, um, you know, indicators for health so, it's um high-risk population. (Participant \#5)

I don't think management gets it, um, I really don't think that they see the level of care that we're providing, um, I'm not sure why. (Participant \#2)

I get the medical complexity, like nobody ever said that but that's kind of how it tends to spell out right, what the doctors don't want to deal with, the NP gets. (Participant \#10)

The NPs also cited that having a great deal of administrative work creates time constraints and impacts the clinical care they provide. "The amount of paperwork is overwhelming and I've had talks with the other staff and said like it's ridiculous like you guys have to do something"..."Right because we have to look at how does this, how does this, um, affect your occupation." (Participant \#3)

NPs were often left to strategize on their own about how to address the added workload burden. "I'm reigning it in."(Participant \#7 sees more patients per day than the physician and sees more socially complex patients). The toll in working with complex, vulnerable populations was evident. "I think it's very rewarding um, but I'm getting really, really tired. It's definitely a shelf life to working like this uh, it's mentally exhausting." (Participant \#5) 


\section{Exploitation of the NP}

The NPs pointed out policies which exploit their practice and negatively impact their ability to grow toward their own potential. For example, there are some funding models where patients are rostered to physicians for which the physician receives corresponding remuneration, but where NPs are cited as the most responsible providers. In some circumstances the NP's purpose is seen solely in their potential to augment physician's income and practice. "Would you just get along with the physicians a little bit? Can you just not make sure you collaborate more so that they protect their numbers?" (Participant \# 4)

Despite the funding physicians receive for patients in some models of care, they may offer very little support to the NPs.

I do practically everything, all ages, all people. Um, I have about 800 rostered patients, they are co-rostered to a physician but the physician really doesn't want anything to do with them. They'll say to you 'those are your patients don't mess up'. (Participant \#7)

In some circumstances, there may be very little consultation with the NP about their level of competency and comfort level in taking on a specific workload. In addition, some NPs are expected to take on roles that are beyond the boundaries of their practice, for which physicians have mechanisms for remuneration, but NPs do not. For example, one primary health care NP was pressured to take on hospital privileges. Not only would this require her to learn a different set of health care knowledge, it would be in addition to her regular practice.

... the problem is I feel I'm working so much already in my primary practice...so I feel I'm already working more than my thirty-seven and a half hours (per week) in primary care. So, I don't know how I'd fit that into my life. (Participant \# 8)

There were circumstances NPs were expected to take on more than they thought they could manage at their current level of expertise and skill "Ultimately, you know, it gets down to your license right, you live in fear that at some point you'd make a mistake or something like that, right?” (Participant \#7)

Policies that reduce the NP's control over their practice also impact their ability to achieve maximum practice potential. One example is scheduling of patients and new intakes where NPs may not be able to screen and make decisions about when to take on a patient, or whether the patient's needs were a good fit with their current practice.

Um, it's a bit of a mystery as far as process goes in our clinic as to who is picked from the waiting list...I've asked my manager several times, like who is deciding, um, because what's happening right now is basically, uh, you know, I could go into my schedule this afternoon and see an intake, and uh, that's just the way it is; this is my new patient and I proceed with the intake... There's one actual situation where I 
received a patient who was really unstable.. to take him on as an intake and I was a little pissed off; because, you know, no one gave me a heads up, no one consulted me to say... you know this is the situation...do you feel like you have the ability to take this patient on?...There's nothing like that....But as far as the number of samedays that are carved out of my schedule and routine appointments, that's all dictated by management. (Participant \#2)

Another comment broadens this discussion:

Um, I guess maybe that segues into another part of this discussion then, so um, so, how much of your practice then is..uh, I don't want to say dictated, but um, determined is probably a better word, because they obviously don't get involved, but by determined by the people, the admin, um, executive director, or whoever's in charge, the board of directors...(Participant \#2).

Figure 1. Evolution of Nurse Practitioner Practice Theory

\section{Maximum Practice Potential}

Scope of practice is not a list of legislated tasks and skills. Rather, it's a reflection on the boundaries of clinical practice. It's a continuum. It's practicing to maximum clinical potential to meet client's health needs. There are both intrinsic and extrinsic factors impacting the NP's ability to practice to their maximum potential.

\section{Intrinsic Factors}

\section{Ability to achieve maximum potential}

a. Depends on level of NP's personal motivation to learn and grow in their practice.

b. Purposefully and consciously working toward maximum potential

c. Personal understanding of what practice at maximum potential looks like. Often includes the extent to which NP practice at maximum potential is modeled

\section{Maximum potential is fluid}

a. The focus of practice changes among different environments so the maximum potential of an individual NP is different in different practices.

b. Each NP has their own maximum potential, changed by experiences, life circumstances.

\section{Extrinsic Factors}

\section{Facilitators to achieving maximum practice potential}

a. being the primary care provider/most responsible provider

b. collegial organizational environment

c. support and resources for continuing education opportunities

Barriers to achieving maximum practice potential

a. Unrealistic workload expectations

b. Exploitation of the NP role 


\section{Discussion}

This grounded theory explored the evolution of NP practice among primary health care NPs in Ontario. Highlighted is the expanded definition of scope of practice to that of the NP's ability to reach maximum practice potential. This is in alignment with other studies where NPs viewed their practice in a broader context than the authorization to complete clinical functions (Donelan, 2013).

The Evolution of NP Practice Theory is also similar to Benner's (1984) work, from Novice to Expert, in that NP practice growth is based on experiential and contextual factors. In addition, gaining expertise is a process characterized by experience and time. Unlike Benner, achieving maximum practice potential is not related to meeting steps, or levels. It is more fluid and changes with the NP's level of motivation, workplace culture and patient population.

The NPs identified both intrinsic and extrinsic factors that influenced the ability to reach their maximum practice potential. Within the intrinsic modality, the NP's personal motivation was identified as the most important factor in growing in their practice role. Some NPs expressed frustration in seeing other NPs who did not strive to reach a higher level of clinical practice. The NPs spoke of this related both to their clinic colleagues as well as their perception of NP practice in other health care models. Although not well established in this study, there are hints that the perception that the participant was working toward continuous improvement in clinical practice, while their colleagues were not doing so, had a negative impact on the NP's job satisfaction.

Personal motivation to achieve growth in their practice is a pivotal factor in the theory. This motivation influences the way NPs approach their practice and may even offset the barriers and potential exploitation they experience in some models of care. Future research may also find another link to job satisfaction where "...the challenges of the full scope of NP role maintained their interest in their work and contributed to satisfaction" (Fournier et al., 2019, p 291).

There are similarities with other literature in the extrinsic factors. Other research cites organizational policies and practices, large number of patients with complex socioeconomic needs, the amount of administrative work, and interprofessional relationships, particularly with physicians as either barriers or facilitators to their practice (Torrens et al. 2020). Although similar to research about NP roles, transition to practice and interprofessional relationships, in this context the extrinsic factors were specifically related to the NP's ability to achieve their maximum practice potential.

\section{Recommendations}

We are entering a time where there is an increased interest in the ongoing practice of NPs, in particular how they might be able to reach their maximum practice potential. Several concepts arose from the NP interviews that speak to the development of strategies that will assist the NP in achieving this. One is the idea of mentorship where 
novice NPs are able to practice with and learn from NPs who not only have experience in the role but have been motivated to achieve their best possible level of practice. Mentorship programs are cropping up, including one developed by the Nurse Practitioners' Association of Ontario (Nurse Practitioners' Association of Ontario, 2021). Ongoing modeling of practice will be instrumental in supporting NPs' practice growth.

The next issue is that of NP autonomy over their day-to-day practice. This may include such things as the ability to choose which patients to add to their roster, length of appointments, integration of added responsibilities, and assistance with clinic operations to free them up to complete administrative duties. Having choice about how they wish to expand their practice is also important for NPs. These issues are situation-specific. Again, NP professional organizations have a part to play in addressing health care policies that lead to these issues and to provide individual NPs with support in addressing their own, specific concerns.

\section{Limitations}

The study sample consisted of NPs in predominantly primary health care practice in Ontario. The experiences of NPs from other jurisdictions and healthcare settings may differ from this research. In addition, the researcher in this study is a primary health care NP from Ontario. Even though prior knowledge of the research area is an asset, the researcher must be cautious in interpreting data so as not to unduly influence the results from their own perspective (Corbin \& Strauss, 2015). In this case, participant feedback was useful in helping to reduce bias.

\section{Conclusions}

NPs are well established in health care systems of many countries. Their value is not only in filling in gaps within those systems, but in their ability to achieve their maximum practice potential. The Evolution of NP Practice Theory offers a framework for ongoing research into this phenomenon along with some suggested areas where strategies may be employed to assist NPs in achieving this objective.

\section{References}

Barnes, H. (2015). Exploring the factors that influence nurse practitioner role transition. The Journal for Nurse Practitioners, 11(2), 178-183. doi:10.1016/j.nurpra.2014.11.004.

Benner, P. (1984). From novice to expert. Excellence and power in clinical nursing practice. Addison-Wesley Publishing Company.

Creswell, J. W. (2013). Qualitative inquiry \& research design. Choosing among five approaches. (3rd Ed.). Sage. 
Corbin, J. and Strauss, A., (2015). Basics of qualitative research. Techniques and procedures for developing grounded theory. (4th Ed.). Sage.

Donald, F., Martin-Misener, R., Bryant-Lukosius, D., Kilpatrick, K., Kaasalainen, S., Carter, N., Harbman, P., Bourgeault, I. L. \& Dicenso, A. (2010). The primary healthcare nurse practitioner role in Canada. Nursing Leadership, 23(sp), 88-113. doi $10.12927 /$ cjnl.2013.22271

Donelan, K., DesRoches, C. M., Dittus, R., and Buerhaus, P. (2013). Perspectives of physicians and nurse practitioners on primary care practice. New England Journal of Medicine, 369(20), 1898-1906. doi:10.1056/NEJMsa1212938

Fournier, J., Lightfoot, N., Larocque, S., Johnson, J. \& Eger, T. (2019). Theory of nurse practitioner job satisfaction. The Journal for Nurse Practitioners, 15(4), p 290-294. https://doi.org/10.1016/j.nurpra.2018.10.011

Hart, A. \& Bowen, A. (2016). New nurse practitioners' perceptions of preparedness for and transition into practice. The Journal for Nurse Practitioners, 12(8), 545-552. Doi https://doi.org/10.1016/j.nurpra.2016.04.018

Hurlock-Chorostecki, C., Forchuk, C., Orchard, C., van Soeren, M. \& Reeves, S. (2014). Hospital-based nurse practitioner roles and interprofessional practice: A scoping review. Nursing and Health Sciences, 16, 401-410. doi: 10.1111/nhs.12107

Kilpatrick., K., Harbman, P., Carter, N., Martin-Misener, R., Bryant-Lukosius, D., Kaasalainen, S., \& Bourgeault., I. (2010). The acute care nurse practitioner role in Canada. Nursing Leadership, 23, (Special Issue) 114-139. $10.12927 / \mathrm{cjn} 1.2010 .22272$

Kleinpell, R. M., Hudspeth, R., Scordo, K. A. \& Magdic, K. (2012). Defining NP scope of practice and associated regulations: Focus on acute care. Journal of the American Academy of Nurse Practitioners, 24(1), 11-18. doi:10.1111/j.17457599.2011.00683.x

Nurse Practitioners' Association of Ontario. (2021). Kintsugi Mentorship Program Information Sheet. Retrieved from: https://mcusercontent.com/fdcb9b07a4a2ad7564aa46b5d/files/6fc16e32-26b84682-8940bd1bc331d20e/Kintsugi_Mentorship_Program_Information_Sheet.01.pdf

Shuler, P. A. \& Davis, J.E. (1993a). The Shuler nurse practitioner practice model: A theoretical framework for nurse practitioner clinicians, educators, and researchers, part 1. Journal of the American Academy of Nurse Practitioners, 5(1), 11-18. https://doi.org/10.1111/j.1745-7599.1993.tb00835.x 
Shuler, P. A. \& Davis, J.E. (1993b). The Shuler nurse practitioner practice model:

Clinical application, part 2. Journal of the American Academy of Nurse

Practitioners, 5(2), 73-88. 10.1111/j.1745-7599.1993.tb00847.x

Torrens, C., Campbell, P., Hoskins, G., Strachan, H., Wells, M., Cunningham, (2020). Barriers and facilitators to the implementation of the advanced nurse practitioner role in primary care settings: A scoping review. International Journal of Nursing Studies, 104(103443). doi: 10.1016/j.ijnurstu.2019.103443. 